\title{
Assessing the innervation of the dorsal wrist capsule using modified Sihler's staining
}

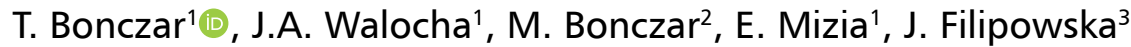 \\ ${ }^{1}$ Department of Anatomy, Jagiellonian University Medical College, Krakow, Poland \\ IIntermed Medical Clinic, Zabierzow, Poland \\ ${ }^{3}$ Department of Translational Research and Cellular Therapeutics, City of Hope, Duarte, CA, USA \\ [Received: 2 January 2020; Accepted: 11 February 2020]
}

\begin{abstract}
Background: The aim of this study was to assess the innervation of the dorsal articular capsule of the wrist using modified Sihler's staining.

Materials and methods: Thirty dorsal wrist capsules were collected from 15 donors (both sides) within 12 hours of death. All the capsules were collected in the same manner - using the dorsal incision. The specimens were stained according to the protocol of the modified Sihler's staining technique. The preserved capsules were analysed under 8-16× magnification of an optical microscope for the presence of major nerve trunks, their major and minor branches, and nerve connections. Results: The range of innervation visualised was that the posterior interosseous nerve innervated approximately $60 \%$ of the central part; the remaining area was innervated by the dorsal sensory branch of the radial nerve and medial antebrachial cutaneous nerve. The constant findings were the branches departing from the ulnar side of the posterior interosseous nerve and from the radial side, with an exception seen in 2 cases. A communicans branch between the posterior interosseous nerve and medial antebrachial cutaneous nerve was seen in all the specimens. The posterior interosseous nerve innervation extended beyond the level of the carpometacarpal joints II-V.

Conclusions: The modified Sihler's staining technique allows for transparent visibility of the nerves that innervate the dorsal wrist capsule. However, it does not allow as accurate assessment as does histological examination, especially regarding the evaluation of nerve endings. Nevertheless, this method provides a significantly larger area of nerve observation than is provided by histological examination. (Folia Morphol 2021; 80, 1: 81-86)
\end{abstract}

Key words: wrist innervation, Sihler's staining technique, posterior interosseous nerve, hand surgery, wrist surgery

\section{INTRODUCTION}

The innervation of the wrist has been a topic of investigation in recent years due to the growing knowledge of wrist pathologies and their treatment. Studies on nerve endings, such as mechanoreceptors, have demonstrated additional important functions of the wrist ligaments in addition to pure stabilisation [8].
However, the extent of innervation of the wrist by individual nerves is yet unclear. Initially, the range of innervation of the wrist was based on the examination of the nerves and their branches with $3.5 \times$ magnification, which was then replaced by microdissection using a microscope. Histological examinations of the stained wrist capsule and ligaments have revealed 
several features in the area of innervation that are in contrast to the findings of microdissections [6]. Microdissection has made it possible to visualise nerve branches of much smaller sizes and their penetration of the joints within the wrist. The purpose of this study was to assess the nerves within the dorsal wrist capsule using the modified Sihler's staining technique.

\section{MATERIALS AND METHODS}

Thirty dorsal wrist capsules were collected from 15 donors (capsules from both sides) within 12 hours of death. There were 11 male ( 22 capsules) and 4 female (8 capsules) wrists. The age range at death of these cadavers was 33-72 years (mean age 65.7 years). Donors whose death was caused by polytrauma with associated upper limb injuries were excluded from the study. The limbs were also checked for the presence of postoperative scars in the wrist and forearm region, both on the dorsal and palmar sides. If scars were present, the wrist joint capsules were not collected.

A longitudinal skin incision was made from the proximal $1 / 3^{\text {rd }}$ of the distal forearm to the middle of metacarpal bone III on the dorsal side. The extensor retinaculum was completely dissected, exposing the dorsal wrist joint capsule. The distal cutting line of the capsule was continued along $1 \mathrm{~cm}$ above the bases of metacarpal bones II-V, proximally along the edges of the distal end of the radius and ulna; the capsule was detached from the periosteum and dorsal radio-ulnar ligament. A fragment of about $1 \mathrm{~cm}$ of the posterior interosseous nerve (PIN) was excised along with its penetration into the capsule.

The lateral and medial edges were determined by the lateral margin of metacarpal II and medial margin of metacarpal V, respectively. The capsules were excised with the extrinsic ligaments detached off its insertions onto the dorsum of the bones of the proximal and distal rows with $3.5 \times$ loupe magnification. The side of capsule excision was noted. The proximal part of the excised capsule was determined by presence of the PIN fragment. The specimens were washed under running water and then fixed in a $10 \%$ un-neutralised formalin solution for a period of 4 weeks.

The following protocol of staining was applied after 4 weeks of fixation in formalin [22]. Maceration in $3 \%$ aqueous potassium hydroxide $(\mathrm{KOH})$ solution for 3 weeks, Sihler's solution I (1 volume glacial acetic acid, 1 volume glycerin, and 6 volumes $1 \% \mathrm{w} / \mathrm{v}$ aqueous chloral hydrate) for staining, Sihler's solution II (1 volume stock Ehrlich's haematoxylin, 1 volume glyc- erin, and 6 volumes $1 \% \mathrm{w} / \mathrm{v}$ aqueous chloral hydrate) for detaining - each phase for 3 weeks. Lithium carbonate solution $0.05 \%$ was then used for neutralisation for 1-2 hours. Finally, dilute $50 \%$ glycerin was used for 3 days for clearing the overstained regions (Won et al. 2011 [22]) and all specimens were preserved with $100 \%$ glycerin [18]. The preserved capsules were analysed under 8-16× magnification with an optical microscope for the presence of major nerve trunks, their major and minor branches, and nerve connections. The obtained image from the microscope was manually applied to the joint capsule diagram, divided into main quadrants and smaller squares with the wrist (right, left) and sides (radial, ulnar) marked. Each quadrant sustained the 256 small squares (16 rows in 16 columns). Thus the total number of squares in 4 quadrants was 1024 . The drawing obtained by hand was scanned and applied to a computer image with accurate reproduction. The area of the innervation of dorsal capsule was determined by two lines running parallel to the long axis of the joint capsule, defined by the outer borders of the furthest reaching and visible nerve branches. Based on the number of squares occupied by the branches of individual nerves, it was possible to calculate the percentage of the area of the joint capsule innervated by a given nerve. The design of this study was approved by our Regional Ethical Review Board.

\section{RESULTS}

The nerves within the 30 wrist capsules in terms of dimensions and course, the number of main trunks and smaller nerve branches, the place of their crossing and the number of small nerves forming the dense network differed in all specimens. In all specimens, the PIN with its branches dominated and covered about $60 \%$ of the surface of the articular capsule. This area was determined by two lines running parallel to the long axis of the joint capsule, defined by the outer borders of the furthest reaching and visible PIN branches. All the PINs had at least one large branch on the ulnar side, but most of them had two larger branches (63.3\%). Three branches on the ulnar side were visible in 7 (23.3\%) cases. Branches on the radial side of the nerve represented similar proportions, although no larger trunks on the radial side were found in two of the 30 specimens. In both these cases, the main trunk or two nerve trunks were clearly passing radially (Fig. 1). Mostly there were two $(43.3 \%)$ branches, but the total share of the 


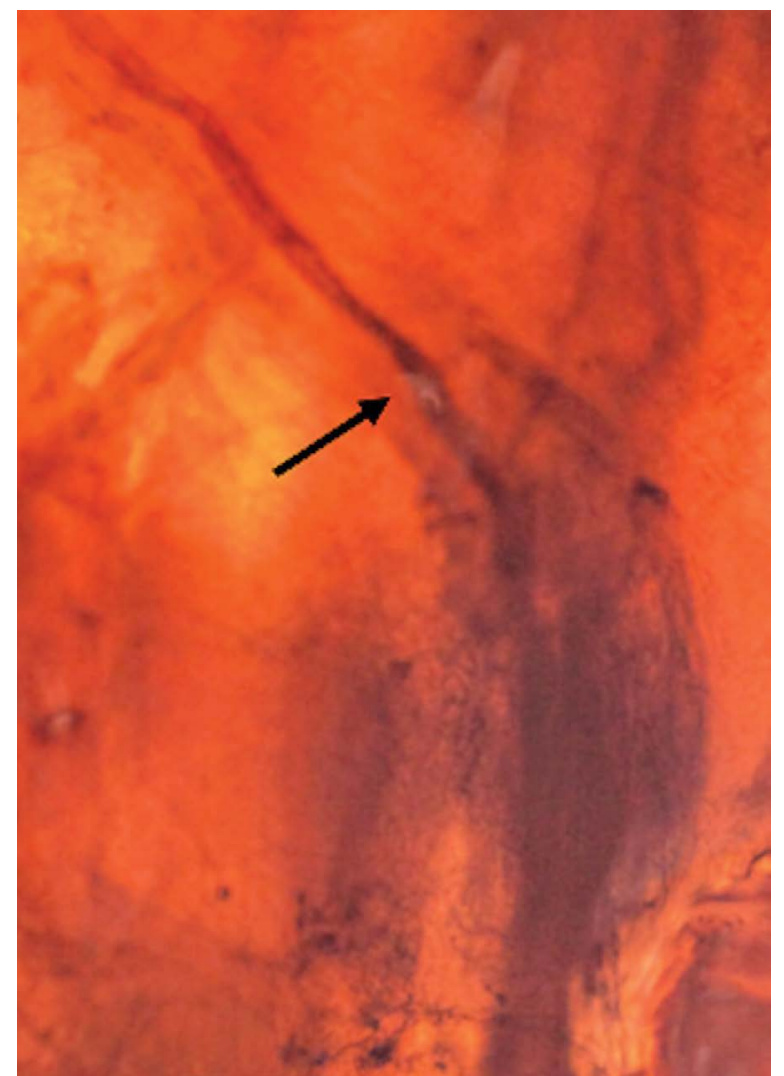

Figure 1. Dorsal wrist capsule (right hand) in Sihler's staining technique. Main trunk of the posterior interosseous nerve (arrow) has no branches on the radial side and turns toward the radial side of the capsule.

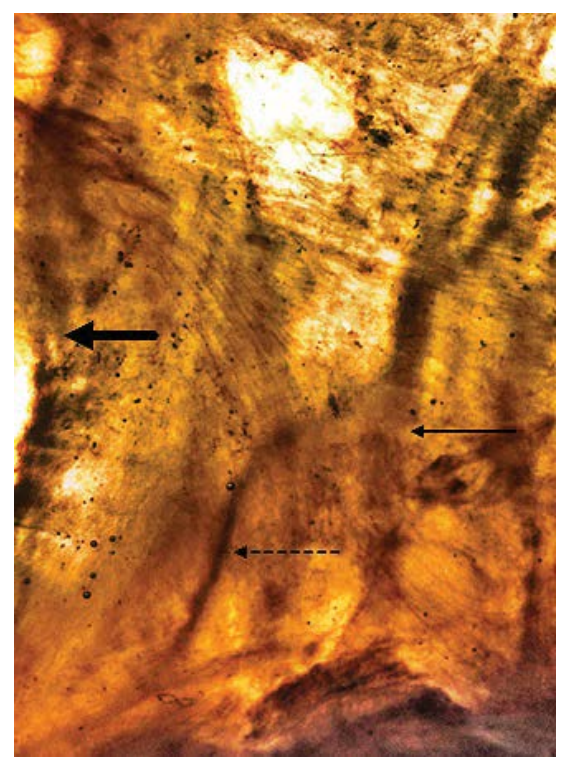

Figure 2. Dorsal wrist capsule (right hand) in Sihler's staining technique. Main trunk of the posterior interosseous nerve (thick arrow), main trunk of the medial antebrachial cutaneous nerve (thick arrow). Communicans branch between both nerves (dotted arrow). number of nerve trunks with 3 or 4 radial branches was identical (43.3\%). In all cases, one or two small branches were visible, protruding from the nerve on the ulnar side, which were directed towards the ulnar and distal direction. It was considered that they were branches connecting to the medial antebrachial cutaneous nerve (MACN), because in the 19 capsules wherein the MACN nerve trunks were clearly visible, these branches were communicating with the main trunk of the MACN. The direction of those branches was always the same. Moreover, in all capsules, there were visible branches that crossed the distal cutting line, in an amount of 1-5. If there was one branch ( 2 cases; $6.7 \%$ ), it ran centrally or slightly radially. In the case of two branches (8 cases; $26.6 \%$ ), they constituted the symmetrical endings of the PIN nerve trunk. In the remaining cases, when there were 3, 4, or 5 branches ( 20 capsules in total; $66.7 \%$ ), they were branches of the main trunk or its larger branches.

All specimens showed a small nerve mesh that occurred symmetrically in 2 (6.7\%) cases. In 16 capsules, a larger number of small nerves were on the radial side of the PIN (78.9\%); in the remaining 12 (40\%) cases, they were on the ulnar side. In the 19 cases where the MACN was visible, its trunk was slightly smaller than or equal to the PIN. In 2 cases where the PIN had two main trunks, the MACN was larger (Fig. 2). In 4 (21.1\%) cases, two MACN trunks were present; in the remaining ones, there was one main trunk (15 cases; $78.9 \%$ ). No major nerve trunks running on the radial border of the PIN were seen. However, in 15 (78.9\%) cases, larger branches crossing the radial cutting line were clearly visible, although they did not show any visible connections with PIN branches. These branches, probably belonging to the superficial branch of the radial nerve (RNSB), were found in numbers of 2 in 8 capsules (53.3\%) and 3 in 2 capsules (13.4\%). In the remaining 5 (33.3\%) cases, there was a single larger branch on the same side. The obtained results are presented in Tables 1-3.

\section{DISCUSSION}

The extent of innervation of the wrist by individual nerves is yet unclear. This is likely because the extent and efficacy of the anatomical examinations keeps changing over time. Initially, the range of innervation of the wrist was based on the preparation of nerves and their branches with $3.5 \times$ magnification, which was then replaced by microdissection using a microscope. Histological examinations of the wrist 
Table 1. Quantitative analysis of the interosseous nerve and its branches based on measurements of 30 joint capsules

\begin{tabular}{|c|c|c|}
\hline Nerve & Amount & Percentage \\
\hline Posterior interosseous nerve & 30 & $100 \%$ \\
\hline Radial branch & 28 & $93 \%$ \\
\hline 0 & 2 & $6.70 \%$ \\
\hline 1 & 2 & $6.70 \%$ \\
\hline 2 & 13 & $43.30 \%$ \\
\hline 3 & 6 & $20 \%$ \\
\hline 4 & 7 & $23.30 \%$ \\
\hline Ulnar branch & 30 & $100 \%$ \\
\hline 1 & 4 & $13.30 \%$ \\
\hline 2 & 19 & $63.30 \%$ \\
\hline 3 & 7 & $23.30 \%$ \\
\hline $\begin{array}{l}\text { Terminal branches (crossing distal } \\
\text { line incision) }\end{array}$ & 30 & $100 \%$ \\
\hline 1 & 2 & $6.70 \%$ \\
\hline 2 & 8 & $26.60 \%$ \\
\hline 3 & 7 & $23.30 \%$ \\
\hline 4 & 8 & $26.70 \%$ \\
\hline 5 & 5 & $16.70 \%$ \\
\hline Majority of small branches side & $\begin{array}{l}\text { Very numerous } \\
\text { nerve networks }\end{array}$ & $100 \%$ \\
\hline Equal & 2 & $6.70 \%$ \\
\hline Radial & 16 & $53.30 \%$ \\
\hline Ulnar & 12 & $40 \%$ \\
\hline
\end{tabular}

capsule stained using the S-100 method [20] revealed several conflicting features with what was found in microdissections. This was particularly relevant for the course of the small nerve branches that are invisible during anatomical preparation, the presence of connections between individual nerves, and the places of entry of the nerves into the joint capsule or wrist joints. The exact scope of innervation of the wrist was particularly important in wrist denervation procedures, which are symptomatic treatments for patients experiencing pain originating from a joint. To avoid undesired denervation of other structures, the knowledge of the specific innervation of the joint was essential.

Sihler's stain is a whole mount nerve staining technique that renders other soft tissues translucent or transparent while staining only the nerves [18]. Wu and Sanders $[24,25]$ were the first to apply the modified Sihler's staining technique to investigate the supply patterns of the laryngeal nerves. Liu et al. [16]
Table 2. Medial antebrachial cutaneous nerve was present in 19 of 30 wrist capsules. There was constant communicants branch departing from ulnar side of the posterior interosseous nerve running in ulnar and distal direction

\begin{tabular}{lcc}
\hline Nerve & Amount & Percentage \\
\hline Medial antebrachial cutaneous nerve & 19 & $63.30 \%$ \\
1 & 15 & $78,90 \%$ \\
2 & 4 & $21.10 \%$ \\
Ramus communicans & 30 & $100 \%$ \\
\hline
\end{tabular}

Table 3. Main trunk of the radial sensory branch was not seen in any specimens. Quantitative analysis of radial branches passing the radial border of the capsule, with no visible connection with branches of posterior interosseous nerve

\begin{tabular}{lcc}
\hline Nerve & Amount & Percentage \\
\hline Superficial branch of radial nerve & 0 & $0 \%$ \\
Ramus on the radial border & 15 & $43.30 \%$ \\
1 & 5 & $33.30 \%$ \\
2 & 8 & $53.30 \%$ \\
3 & 2 & $13.40 \%$ \\
\hline
\end{tabular}

were the first to use Sihler's stain to determine the intramuscular nerve supply of the long head of the triceps muscles in rabbits. Several further studies on the innervation of limb and trunk muscles in humans and monkeys were then carried out in subsequent years [11-14, 23]. Using Sihler's stained human foetal specimens, Peker et al. [19] investigated the relations between the shape of skeletal muscles and their nerve distribution patterns. To our knowledge, the modified Sihler's staining technique has never been used to qualitatively assess the course of nerves in the wrist capsule. Our study enabled the visualisation of nerve branches inside the joint capsule that would not be visible during dissection, even at high magnification.

Many studies show that the main innervation of the wrist from the dorsal comes from the PIN [1-3, $6-10,15,21]$. All the above mentioned studies were based on the preparation of nerve branches or those visible during surgery. In our study, it was possible to determine the range of innervation ratio of the entire joint capsule based on the actual image. Approximately $60 \%$ of the central part of the capsule is supplied by the PIN, radial part by the RNSN (approximately $15 \%$ ) and the MACN innervates approximately $25 \%$ of the capsule from the ulnar side. However, the technique we used does not allow us to determine 


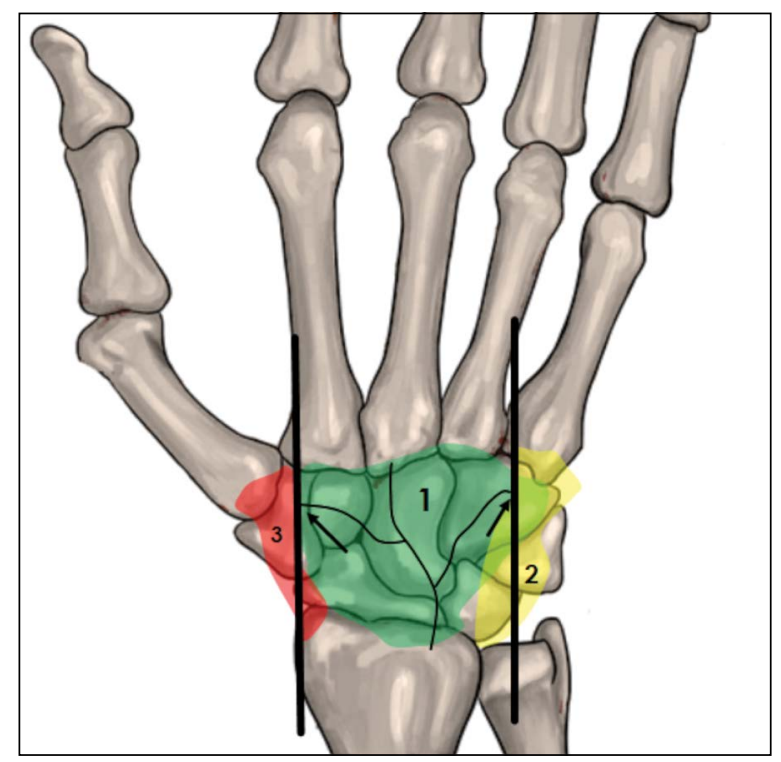

Figure 3. Innervation of dorsal aspect of wrist joint. This drawing presents a global overview of innervation and does not show the exact range and possible overlapping. The area of posterior interosseous nerve innervation was determined by two lines running parallel to extend the long axis of the joint capsule, defined by the outer borders of the furthest reaching and visible posterior interosseous nerve branches (arrows); 1 - posterior interosseous nerve innervation area; 2 - dorsal branch of the ulnar nerve innervation area; 3 - superficial branch of radial nerve innervation area.

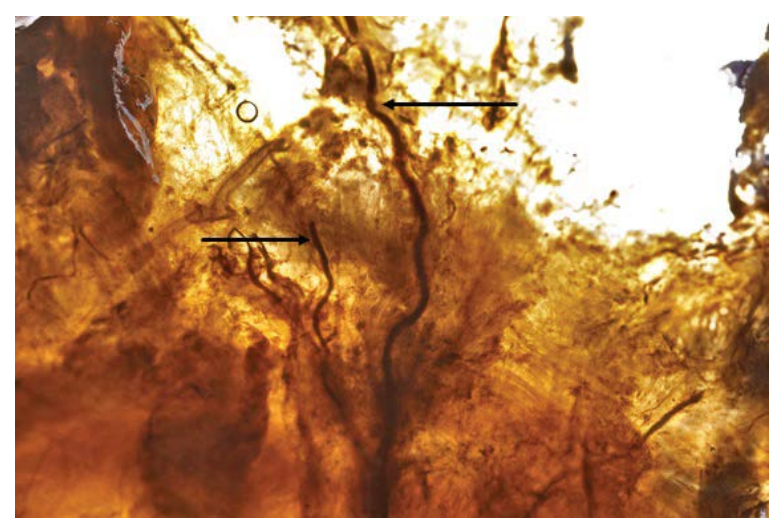

Figure 4. Dorsal wrist capsule (left hand) in Sihler's staining technique. Two terminal branches (arrows) of the posterior interosseous nerve crossing the distal border (white area) of the capsule.

where the innervation ranges overlap (Fig. 3). Another important finding of this study is that the terminal branches crossing the distal line incision were found to be fairly consistent; the incision was performed $1 \mathrm{~cm}$ distal to the carpometacarpal (CMC) joints II-V (Fig. 4).

Only in 2 of the 30 specimens was single branch noted; in the remaining specimens, the number of branches varied from 2 to 5 , on average 3.6 per capsule. In our specimens, these branches were crossing the cutting line, which did not show the presence of the small end branches. We therefore reached the conclusion that these are not the branches ending in and innervating the CMC joints. Dellon et al. $[4,5]$ dissected the PIN and its branches in 10 cadavers; they showed that in 2 of the 10 cadavers, the terminal branches of the PIN appeared to extend to the metacarpophalangeal joints, traveling in the deep fascia overlying the interosseous muscles. The methodology of our study did not allow the conclusion that all of these branches innervate the metacarpophalangeal joints, but the number of PIN nerve branches that run through the metacarpals was undoubtedly greater. McCarthy and Breen [17] showed that 14 of 41 $(34 \%)$ specimens in which the $3.5 \times$ magnification of PIN dissection was performed, had innervations of terminal branches that included the CMC joints, the interossei, and the metacarpal periosteum. They also observed three levels of innervation that were present in most specimens: radiocarpal, midcarpal, and terminal branches. However, only fourteen of their specimens had innervations at all three levels. The present study shows that range of PIN innervation reaches beyond the CMC joint's line, which is more distal than the central two-thirds of the posterior wrist joint; this is similar to what was reported by Lin et al. [15].

We realise that there are several limitations to this technique of staining. This method does not enable distinction between sensory and motor neurons, provides less detailed information concerning the relations of the nerve terminal structures, and is time consuming. In addition, differences in staining quality may be seen depending on the thickness and size of the specimen as well as consequent to inaccurate solution changes during the decalcification and clearing steps [18]. However, in our opinion, it can be considered as an interesting supplement to the assessment of the nerves within the wrist capsule along with previously used methods of preparation or histological staining.

\section{CONCLUSIONS}

Modified Sihler's staining allows the clinician to obtain a wider picture of the range of innervation of the wrist joint capsule, much larger than when preparing individual nerves. It provides the opportunity to visualise nerves with much smaller cross sections, smaller than those obtained during microdissection. However, it does not allow assessment as accurate as 
that possible with histological examination, especially in evaluation of the nerve endings, but it provides a significantly larger area of nerve observation.

\section{REFERENCES}

1. Apergis E. Wrist anatomy in: Fracture-Dislocations of the Wrist. Springer 2013: 35-37.

2. Braga-Silva J, Román JA, Padoin AV. Wrist denervation for painful conditions of the wrist. J Hand Surg Am. 2011; 36(6): 961-966, doi: 10.1016/j.jhsa.2011.03.004, indexed in Pubmed: 21549522.

3. Dellon AL. Partial dorsal wrist denervation: resection of the distal posterior interosseous nerve. J Hand Surg Am. 1985; 10(4): 527-533, doi: 10.1016/s0363-5023(85)80077-0, indexed in Pubmed: 4020064.

4. Dellon A, Mackinnon S, Daneshvar A. Terminal branch of anterior interosseous nerve as source of wrist pain. J Hand Surg. 1984; 9(3): 316-322, doi: 10.1016/02667681(84)90051-2.

5. Dellon A, Seif S. Anatomic dissections relating the posterior interosseous nerve to the carpus, and the etiology of dorsal wrist ganglion pain. J Hand Surg Am. 1978; 3(4): 326-332, doi: 10.1016/s0363-5023(78)80032-x, indexed in Pubmed: 681715.

6. Ferreres A, Suso $S$, Ordi J, et al. Wrist denervation. Anatomical considerations. J Hand Surg. 2016; 20(6): 761-768, doi: 10.1016/s0266-7681(95)80043-3.

7. Grechenig W, Mähring M, Clement HG. Denervation of the radiocarpal joint. J Bone Joint Surg. British Volume. 1998; 80-B(3): 504-507, doi: 10.1302/0301-620x.80b3.0800504.

8. Hagert $E$, Forsgren $S$, Ljung BO. Differences in the presence of mechanoreceptors and nerve structures between wrist ligaments may imply differential roles in wrist stabilization. J Orthop Res. 2005; 23(4): 757-763, doi: 10.1016/j. orthres.2005.01.011, indexed in Pubmed: 16022987.

9. Hagert E, Garcia-Elias M, Forsgren S, et al. Immunohistochemical analysis of wrist ligament innervation in relation to their structural composition. J Hand Surg Am. 2007; 32(1): 30-36, doi: 10.1016/j.jhsa.2006.10.005, indexed in Pubmed: 17218173.

10. Hixson ML, Stewart C. Microvascular anatomy of the radioscapholunate ligament of the wrist. J Hand Surg Am. 1990; 15(2): 279-282, doi: 10.1016/0363-5023(90)901084, indexed in Pubmed: 2324457.

11. Hua J, Kumar VP, Pereira BP, et al. Split flexor carpi radialis muscle. Plast Reconstr Surg. 1999; 103(3): 930-934, doi: 10.1097/00006534-199903000-00024, indexed in Pubmed: 10077083.

12. Kumar VP, Liu J, Lau HK, et al. Neurovascular supply of the gracilis muscle: a study in the monkey and human. Plast Reconstr Surg. 1998; 101(7): 1854-1860, doi: 10.1097/00006534-199806000-00012, indexed in Pubmed: 9623827.

13. Lim A, Kumar $P$, Hua J, et al. The Neuromuscular Compartments of the Flexor Carpi Ulnaris. Plast Reconstr
Surg. 1999; 103(3): 1046-1051, doi: 10.1097/00006534199903000-00048.

14. Lim AYT, Pereira BP, Kumar VP, et al. Intramuscular innervation of upper-limb skeletal muscles. Muscle Nerve. 2004; 29(4): 523-530, doi: 10.1002/mus.10565, indexed in Pubmed: 15052617.

15. Lin YT, Berger RA, Berger EJ, et al. Nerve endings of the wrist joint: a preliminary report of the dorsal radiocarpal ligament. J Orthop Res. 2006; 24(6): 1225-1230, doi: 10.1002/jor.20166, indexed in Pubmed: 16705705.

16. Liu J, Kumar P, Shen Y, et al. Modified Sihler's technique for studying the distribution of intramuscular nerve branches in mammalian skeletal muscle. Anat Record. 1997; 247(1): 137-144, doi: 10.1002/(sici)10970185(199701)247:1<137::aid-ar16>3.0.co;2-q.

17. McCarthy C, Breen T. Arborization of the distal posterior interosseous nerve. J Hand Surg. 1995; 20(2): 218-220, doi: 10.1016/s0363-5023(05)80010-3.

18. Mu L, Sanders I. Sihler's whole mount nerve staining technique: a review. Biotech Histochem. 2010; 85(1): 19-42, doi: 10.3109/10520290903048384, indexed in Pubmed: 19572223.

19. Peker T, Gülekon N, Turgut BH, et al. Observation of the relationship between the shape of skeletal muscles and their nerve distribution patterns: a transparent and microanatomic study. Plast Reconstr Surg. 2006; 117(1): 165-176, doi: 10.1097/01.prs.0000186539.80555.27, indexed in Pubmed: 16404263.

20. Rhodes RH. Diagnostic Immunostaining of the Nervous System. In: Bennington JL (Ed.). Major Problems in Pathology (vol 19): Immunomicroscopy: A Diagnostic Tool for the Surgical Pathologist. Saunders, Philadelphia 1986: 334-343.

21. Van de Pol GJ, Koudstaal MJ, Schuurman AH, et al. Innervation of the wrist joint and surgical perspectives of denervation. J Hand Surg Am. 2006; 31(1): 28-34, doi: 10.1016/j. jhsa.2005.06.021, indexed in Pubmed: 16443100.

22. Won SY, Kim DH, Yang HM, et al. Clinical and anatomical approach using Sihler's staining technique (whole mount nerve stain). Anat Cell Biol. 2011; 44(1): 1-7, doi: 10.5115/ acb.2011.44.1.1, indexed in Pubmed: 21519543.

23. Wong MT, Lim AY, Coninck CD, et al. Functional units within the latissimus dorsi muscle based on Sihler technique. Ann Plast Surg. 2007; 59(2): 152-155, doi: 10.1097/01.sap.0000252731.60748.cb, indexed in Pubmed: 17667408.

24. Wu BL, Sanders I. A technique for demonstrating the nerve supply of whole larynges. Arch Otolaryngol Head Neck Surg. 1992; 118(8): 822-827, doi: 10.1001/ archotol.1992.01880080044011, indexed in Pubmed: 1379438.

25. Wu BL, Sanders I, Mu L, et al. The human communicating nerve. An extension of the external superior laryngeal nerve that innervates the vocal cord. Arch Otolaryngol Head Neck Surg. 1994; 120(12): 1321-1328, doi: 10.1001/ archotol.1994.01880360019004, indexed in Pubmed: 7980895. 